\title{
Informing mental health policies and services in the EMR: cost-effective deployment of human resources to deliver integrated community-based care
}

\author{
G. Ivbijaro, ${ }^{7}$ V. Patel, ${ }^{2}$ D. Chisholm, ${ }^{3}$ D. Goldberg, ${ }^{4}$ T.A.M. Khoja ${ }^{5}$ and T.M. Edwards, ${ }^{6}$ Y. Enum ${ }^{7}$ and L.A. Kolkiewic ${ }^{8}$
}

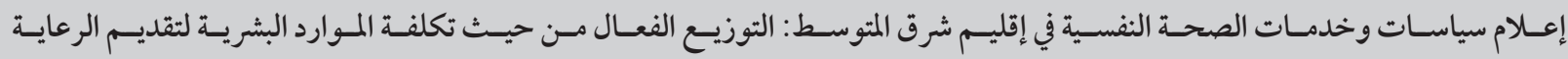

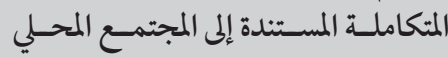

$$
\begin{aligned}
& \text { جبريل إيفيبجارو، فكرام اتل، دانييل شيسهولم، ديفيد جولدبرج، توفيق خوجة، تود إدوارد، يعقوب إينوم، لوتشيا كولكيفيتش }
\end{aligned}
$$

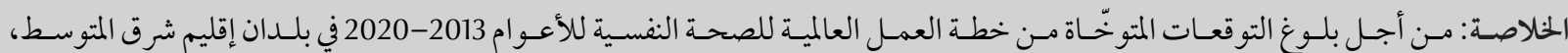

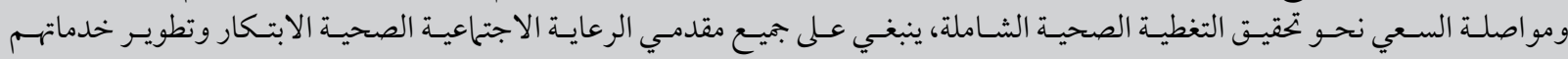

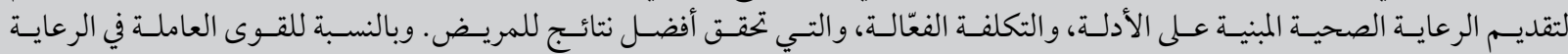

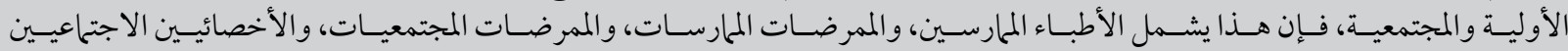

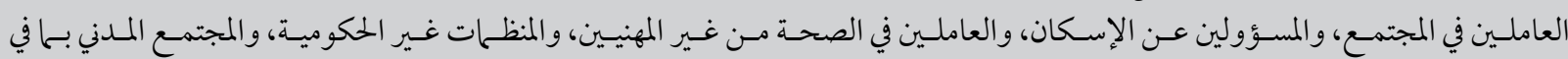

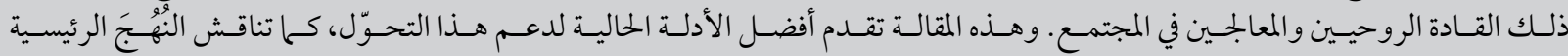

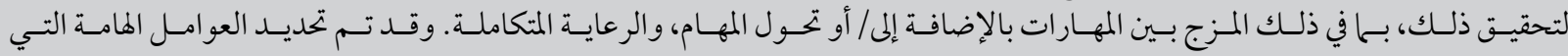

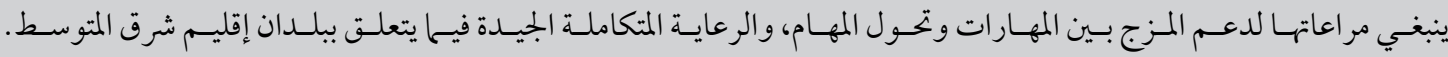

ABSTRACT For EMR countries to deliver the expectations of the Global Mental Health Action Plan 2013-2020 \& the ongoing move towards universal health coverage, all health \& social care providers need to innovate and transform their services to provide evidence-based health care that is accessible, cost-effective \& with the best patient outcomes. For the primary and community workforce, this includes general medical practitioners, practice \& community nurses, community social workers, housing officers, lay health workers, nongovernmental organizations \& civil society, including community spiritual leaders/healers. This paper brings together the current best evidence to support transformation \& discusses key approaches to achieve this, including skill mix and/or task shifting and integrated care. The important factors that need to be in place to support skill mix/task shifting and good integrated care are outlined with reference to EMR countries.

Orienter les politiques et les services santé mentale dans la Région de la Méditerranée orientale : déploiement de ressources humaines d'un bon rapport coût-efficacité pour une prestation de soins communautaires intégrés

RÉSUMÉ Pour que les pays de la Région de la Méditerranée orientale puissent répondre aux attentes créées par le Plan d'action mondial sur la santé mentale 2013-2020 et pour faciliter le mouvement continu vers la couverture sanitaire universelle, tous les acteurs de la prestation de soins socio-sanitaires doivent faire preuve d'innovation et transformer leurs services afin de fournir des soins de santé fondés sur des bases factuelles qui soient accessibles, d'un bon rapport coût-efficacité et procurent les meilleurs résultats pour les patients. Pour ce qui est des personnels aux niveaux primaires et communautaires, ceci concerne les médecins généralistes, les infirmières praticiennes, les infirmières communautaires, les travailleurs sociaux communautaires, les responsables des logements sociaux, les travailleurs de la santé non professionnels, les membres des organisations non gouvernementales et de la société civile, y compris les leaders et les guérisseurs spirituels communautaires. Le présent article rassemble les meilleures bases factuelles actuellement disponibles à l'appui de cette transformation et examine les approches principales à cet égard, y compris l'éventail des compétences et/ou la délégation des tâches et les soins intégrés. Les facteurs importants qui doivent être en place à l'appui de l'éventail des compétences/la délégation des tâches et de bons soins intégrés sont présentés dans le contexte des pays de la Région de la Méditerranée orientale.

${ }^{7}$ Wood Street Medical Centre, London, United Kingdom (Correspondence to G. Ivbijaro: gabriel.ivbijaro@gmail.com). ${ }^{2}$ Department of International Mental Health, London School of Hygiene and Tropical Medicine, London, United Kingdom \& Centre for Chronic Conditions and Injuries, Public Health Foundation of India, New Delhi, India. ${ }^{3}$ Department of Mental Health and Substance Abuse, World Health Organization, Geneva, Switzerland. Institute of Psychiatry, King's College, London, United Kingdom. ${ }^{5}$ General Executive Board, Health Ministers Council for Cooperation Council, Riyadh, Saudi Arabia. (Continued on page 412) 


\section{Case for transforming mental health services in the EMR}

The countries of the Eastern Mediterranean Region (EMR) continue to undergo rapid social and cultural changes, which bring both advantages and challenges. For countries in the Region to meet the expectations of the World Health Organization's (WHO) Global Mental Health Action Plan 2013-2020 (1) and to progress towards universal health coverage (2), all those involved in the provision and delivery of health and social care need to innovate and transform their services so that these deliver evidence-based health care that is accessible and cost-effective and has the best patient outcomes.

The Mental Health Atlas 2011 describes resources for mental health in the EMR and shows large variations in healthcare expenditure, manpower and health systems delivery among the Member States of the EMR (3). This is reflected in differing life expectancies and literacy rates and variations in the contribution of neuropsychiatric disorders to the national burdens of disease. There is also variability in governance across the countries of the Region in terms of mental health policy, mental health plans, mental health legislation and mental health financing. There is a need to learn from current best practice within the EMR and similar health economies to ensure that individual countries, independent of the resources available to them, can work towards achieving best practice.

Challenges shared by all nations of the Region, regardless of income, are a shortage of medical manpower (including a shortage of psychiatrists, general medical practitioners, social workers, psychologists, occupational therapists and mental health nurses); a lack of integrated primary care services; and variable access of patients to psychotropic medication, due to differences among countries in prescribing rights of secondary and primary care personnel.
With improving medical technology many people are likely to live longer and with increasing age there will be increasing morbidity and multi-morbidity. It needs to be recognized that mental and physical health co-morbidity will continue to be the norm rather than the exception.

A recent review of projections for global mortality and burden of disease shows that noncommunicable diseases and mental health will continue to be the leading causes of mortality and morbidity in low-, medium- and high-income countries. (4) There is increasing evidence that noncommunicable diseases and mental health co-morbidity have an additive effect (5-8) and this suggests that mental health-care skills need to be embedded throughout the primary care workforce. In a review of 23 low- and medium-income countries it has been estimated that if nothing is done to address chronic diseases in developing countries, US\$ 84 billion of economic production could be lost, in addition to the high costs of care to the health systems (9). We need a new primary care workforce that will work in the community, utilizing all evidence of best practice obtained from low, mediumand high-income countries (9-11).

Global changes in patterns of morbidity also provide opportunities for innovation and new ways of working. In order to achieve the benefits of universal health coverage there is a need to develop a comprehensive approach to care, with clearer pathways and with a defined role for the state, for health commissioners, hospital health providers, primary care providers, nongovernmental organizations, civil society and other community health and social care providers. There is a need to recognize the role of the individual patient and his/her carers in self-care and health promotion.

Despite the shortage of specialists in low- and medium-income countries of the EMR, there are effective treatments for common mental health problems that can be applied in a primary care setting. This also makes it possible to address co-morbidity, to improve access to mental and physical health care and use a patient-centred approach, which ultimately reduces the stigma of mental illness $(7,10,12)$. One of the key innovations required is to develop and deliver an appropriately skilled workforce within the primary care system, through skill mix (13-16) and/or task-shifting (17-19) supported by an evidence-based system of collaborative or integrated care (20-22).

\section{Workforce transformation}

The impact and financial cost of longterm health problems in 23 low- and medium-income countries similar to those in the EMR has been estimated at US\$ 84 billion if nothing is done to address problems such as heart disease, stroke and diabetes (6-9). When mental health co-morbidity is added into the equation, this loss may be as much as $70 \%$ greater. Research shows that integration of mental health care into primary care is effective, cost-effective and safe, and this is true in high-, medium- and low-income countries (20). EMR Member States require a template that can be applied to support integration in their specific health-care setting, while recognizing that each country will have a different starting point depending on the resources and manpower that are already available. Techniques that can be applied to develop the workforce to meet this challenge are skill mix and task shifting.

\section{Defining skill mix and task shifting}

The terms task shifting and skill mix tend to be used interchangeably in the literature, and it is therefore necessary to clarify the terms.

Skill mix can be defined as a group of professionals working together within the same team, each contributing to the 
care of the same individual patient by following a single pathway in an integrated fashion or in a collaborative way using a stepped-care approach $(14-16,20)$. This technique is well-known and welltested in primary care globally and this article will propose how this concept can be applied to mental health-care delivery in the EMR.

Task shifting is a methodology through which activities previously provided by clinicians are transferred to others within or outside the team. There are over 50 years of evidence demonstrating its safety and effectiveness in low-, medium- and high-income countries (20). For example, a randomized controlled trail in India showed that interventions for depressive and anxiety disorder led by lay health workers decreased the prevalence of common mental disorders and resulted in a reduction in suicide attempts (21).

The effectiveness of task shifting and skill mix depends on the quality of the people who are taking on the new roles (19) and there is therefore a need to train the new workforce appropriately.

\section{Skill mix and task shifting-key enablers}

Key steps need to be in place to achieve successful skill mix or task shifting. These include:

- A clear philosophy and initiative which defines the scope of the collaboration or integration. This would include the type of patients or clients who will be managed, the funding arrangements, the infrastructure required and which patients or clients will be excluded from the type of service provided.

- Leadership and clinical champions who will provide the governance, the vision and mission and advocate for a transformed service. They will be responsible for the monitoring and implementation of clinical governance and quality improvements. One way to ensure the engagement of leadership is to set up a board of stakeholders who must include psychiatrists, general practitioners and other members of the primary health-care workforce, patient organizations, community links and other organizations that have an interest in supporting the success of the initiative.

- Appropriate infrastructure and information technology in place to supportachange in working. This would include patient records and data-sharing agreements supported by a legal framework across the patient pathway.

- A well-defined clinical pathway which aligns the different components of the workforce necessary to deliver the pathway. The workforce required can be calculated using disease incidence and prevalence data, the numbers of patients who are already being managed and an estimate of the numbers that will need to be managed to better meet the burden of disease. In order to identify what will be needed from the extended workforce the pathway needs have definitions of the expected parameters of self-care and the nature of the evidence-based interventions required to address the disease burden.

- A supportive legislative or policy environment that supports integration and workforce development. There may need to be changes in legal requirements to ensure that skill mix and task shifting are properly applied and this would include a clear definition of the role enhancements within the workforce. There needs to training in the competencies required by the workforce to perform their new roles, using the principles of patient-centred care and taking into account public health needs and expectations (15).

- A clear policy for the accreditation of a new workforce and clarity of task. This must include a framework for continuous professional development, mentoring schemes, supervision and career progression.

- Expandingthe curriculum of medical and nurse training to include collaborative care, mental health and working within systems. Current core and professional training needs to be transformed to focus on integrated practice and joint working in order to overcome barriers across sectors.

\section{Understanding the new terminology}

New roles and responsibilities brought about through a transformation process often require the introduction of new, culturally appropriate terminology. The terminology adopted to address the issue of changing and evolving clinical roles should make little difference as long as the change is supported by collaborative stepped or integrated care. There is sufficient evidence of the costeffectiveness of this approach in primary and community settings $(12,17,22,23)$.

What is important for successful transformation is that any process that includes skill mix or task shifting and integration is bidirectional. This means that there should be parity between mental and physical health-care delivery to avoid the risk of marginalizing mental health. An example is the need to train midwives and health visitors in mental health-care skills as well as maternity and paediatric skills so that they can play an active role in screening for mental illness and develop appropriate management plans in collaboration with local primary health-care networks to achieve the best outcome for their patients and families. General hospital staff require mental health training and input from local mental health services or from primary care mental health services. Similarly, specialist mental health services require input from physical health services in primary and secondary care.

What patients, their families and carers require is an integrated, simplified pathway supported by appropriate human resources and policies to support this change in practice.

Well-defined skill mix and task shifting also enhances the role of the family doctor, as some of them will become 
general practitioners with a special interest $(\mathrm{GPwSI})$ in primary care mental health (24). Countries of the EMR will require support to transform their primary health-care workforce and to simplify access to such care. This can be achieved by general medical practices adopting a model of primary care networks supported by community and hospital resources.

There are currently variations in prescribing rights of health-care providers and hence in patients' access to medication across the EMR and therefore there will be a need to ensure better access in primary care to psychological therapies and to the range of psychopharmacological interventions. The is also a need to focus on mental health promotion, for example by developing the skills of the general population in mental health first aid, by improved management of long-term physical health conditions (many of which are co-morbid with common mental health conditions) and by improved access to substance misuse treatment interventions (including smoking cessation). This is what is meant by "primary care transformation”.

\section{Strategies for delivering integrated care}

Delivering mental health care in the community and via the primary healthcare system is not achieved just by an increase in number of workers. Primary care transformation requires the existing workforce to work differently and for new workforce roles to be developed (20,21).

An example of this from a highincome country is the use of navigators. A navigator is a single, named individual who can help people with multiple morbidities and those who are vulnerable, especially those with long-term health conditions, to navigate their way through complex systems (e.g. across health, social care, housing, employment and education) and who can help to pull together integrated care packages across sectors and services (25-27).

An analysis of integration in health research, policy and practice noted that system-wide collaboration must go beyond the health sector. It suggested that that the well-being of the most vulnerable health-system users, whose mental and physical symptoms lead to disorders with persistent impairments, may be a sensitive indicator of a society's need for integrated care, and that full social participation for vulnerable groups requires sustained access to jobs, schools and other services. This requires cooperation among the education, social services, labour and justice sectors (27-30)

\section{Key factors supporting good integrated care}

\section{Information-sharing systems}

To support the effective day-to-day provision of integrated care to people with mental health problems it is essential that information systems are compatible within and across different organizations. The system would establish individual electronic records of patients' integrated health and social care needs and interventions; would have the facility to record information about education, housing, welfare benefits and employment status, identifying specific occupational health needs; and would require the ability to anonymize and aggregate health and social care records to inform a needs assessment of the local population and hence local joint and multi-agency commissioning plans.

\section{Shared protocols}

Shared protocols between two or more organizations, or parts of an organization, are needed to set out the responsibilities of each in delivering an agreed service and/or outcome. Where shared protocols have been established, the evidence suggests they work well, although it is important to ensure that staff "buy-in" to the protocol. Shared protocols should be developed within and across the range of statutory, independent and voluntary organizations that support people with mental health problems.

\section{Improved access to primary care psychological and pharmacological therapy}

The effectiveness of improving access to primary care psychological and pharmacological therapy has been demonstrated in the United Kingdom, Ireland, New Zealand and Saudi Arabia. It is hoped that it will reduce some of the intercountry variation across the EMR that was highlighted in the Mental Health Atlas 2011 (3).

\section{Joint funding and commissioning}

Separate funding streams hinder integrated care, while pooled funding and services commissioned across boundaries increase the likelihood of patients receive better care. Combining health, social care and other budgets (e.g. for education) at a local commissioning level provides the opportunity to mirror the service delivery requirements of people who need a single, coordinated approach to manage their mental health condition. Commissioners need to be aware that the support of people with mental health needs extends beyond traditional health and social care interventions, to help with issues related to lifestyle choices such as exercise and smoking.

\section{Co-located services}

The co-location of primary care and specialist mental health staff is strongly supported in the literature and could bring significant benefits to patients in terms of a better integrated response to their needs, so long as the staff understand their respective roles and responsibilities and work collaboratively, and willingly, together. There is no evidence 
that the merging of organizations involved in providing different aspects of care to people with mental health needs would in itself improve that care.

\section{Liaison services}

There are significant benefits to establishing both psychiatric liaison services in physical health-care settings and physical care liaison services in mental health-care settings. Commissioners need to be more aware of the evidence for the effectiveness of such services, the improvements to integrated patient care and the cost savings that can be made.

\section{Navigators or case managers}

There is benefit in having a single, named individual who can help people navigate their way through complex systems across health, social care, housing, employment and education (among other services) and help to pull together integrated care packages. This would go a long way to ensuring that people received effective, integrated care.

\section{Reduction of stigma}

A public and a health-care workforce who are better informed about health and mental health issues would help to create an environment in which a truly integrated response to poor mental health could be established. In addition to continuing work in raising the public's mental health awareness, both primary and secondary schools need to ensure that emotional and mental health issues are fully integrated into what children are taught about health and healthy living in the widest sense. This would mean that young adults intending to move into careers in health and social care already have a basic understanding of the indivisibility of physical and mental health, so that the formal professional training on holistic and integrated care

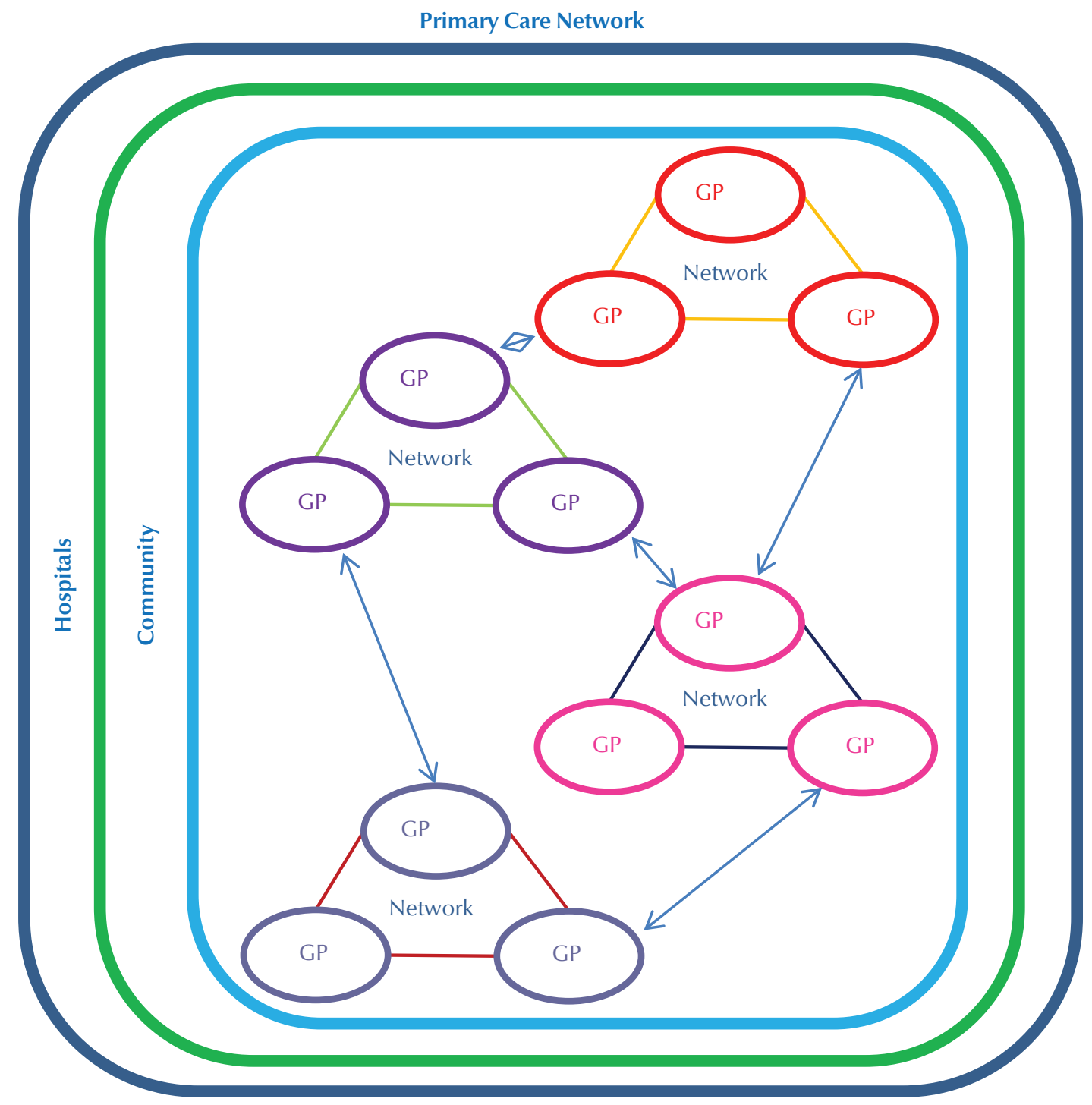

Figure 1 A primary care network represents a geographical area served by general medical practices, community resources and hospital. Each circle represents a general practice working collaboratively with other general practices as a primary care network 
that they receive from day one will come to them quite naturally. By itself, though, this is not enough; there is a small, but good, evidence base suggesting that interpersonal contact involving people with mental health problems can reduce the risk of their suffering stigmatizing attitudes and behaviour from other.

\section{Aligning hospital care to primary care}

An essential component supporting primary care mental health transformation are well-organized mental health hospital and secondary level health services which align with and support the local primary health-care networks (Figure 1).

\section{Conclusion}

To successfully transform mental health services in the EMR it is essential that successful models for integrated services in local areas are scaled up both nationally and regionally in order to provide health and social care benefits to as many individuals in the population as possible. This will contribute to the delivery of the Global Mental Health Action Plan 2013-2020 and universal health coverage.

Leadership across all sectors is an essential component in the delivery of integrated or collaborative mental health care. This requires high-level commitment and collaborative relationships across all grades of health service managers and clinicians.

Some practical steps need to be taken by the EMR Member States, supported by the WHO and other important stakeholders such as the World Organization of Family Doctors (WONCA), World Federation for Mental Health (WFMH) and other academic and health policy institutions to transform primary-care mental health. These are summarized in Table 1.

Table 1 Scaling up primary-care based mental health services in the Eastern Mediterranean Region (EMR) Step 1: Region-specific measures

-Establish a multisectoral regional committee across EMR to:

- articulate the vision across the region and summarize the case for change;

- provide technical expertise;

- share best practice across the Region;

- share resources to avoid duplication;

- coordinate lessons learnt;

- agree operational, governance, financial and performance frameworks.

- Agree an EMR matrix for monitoring performance.

- Agree for co-morbid physical and mental health conditions to be tackled, including diabetes, respiratory conditions and musculoskeletal conditions that are common in primary care.

-Agree a structural framework for data collection that will be used across all sectors for benchmarking.

-Agree the minimum data set to be collated by countries across the whole Region.

- Agree that the population be covered by each primary-care network.

-Identify gaps in the workforce and propose the development of a new workforce.

Step 2: country-specific measures

- Establish a national accountability and joint decision-making group in each Member State to ensure that country-specific issues are covered, including proposed national information technology solutions, the identification of national clinical priorities and definition of population groups per unit.

- Establish a national clinical reference group.

- Agree a national formulary for prescribing.

- Agree pathways for access to psychological therapies.

- Agree geographical limits of each primary-care network.

- Confirm information sharing agreements and proposed incentives.

- Agree an accreditation process for new skills and a method of monitoring safety and clinical pathway fidelity.

- Agree a national matrix for monitoring performance.

- Design and implement incentive schemes to support change.

- Agree the scope of national scaling-up.

- Map all the local resources that can be deployed as part of skill mix. 
References

1. Mental Health Action Plan 2013-2020. Geneva: World Health Organization; 2013.

2. The world health report 2010. Health system financing: the path to universal coverage. Geneva: World Health Organization; 2010 (http://www.who.int/whr/2010/whr10_ en.pdf?ua=1, accessed 18 June 2015).

3. Mental Health Atlas-2011 country profiles [Internet]. Geneva: World Health Organization; 2011 (http://www.who.int/mental_health/evidence/atlas/profiles/en/, accessed 18 June 2015).

4. Mathers CD, Loncar D. Projections of global mortality and burden of disease from 2002 to 2030. PLoS Med. 2006 Nov;3(11):e442. PMID:17132052

5. The global burden of disease: 2004 update. Geneva: World Health Organization; 2008.

6. Katon WJ, Von Korff M, Lin EHB, Simon G, Ludman E, Russo J, et al. The Pathways Study: a randomized trial of collaborative care in patients with diabetes and depression. Arch Gen Psychiatry. 2004 Oct;61(10):1042-9. PMID:15466678

7. Ivbijaro G. Mental health: a resilience factor against both NCDs and CDs. Cambridge (MA): Nexus Strategic Partnerships; 2012. pp. 17-20 (http://www.commonwealthhealth. org/wp-content/uploads/2012/05/17-201.pdf, accessed 18 June 2015)

8. Ivbijaro GO, Enum Y, Khan AA, Lam SS, Gabzdyl A. Collaborative care: models for treatment of patients with complex medical-psychiatric conditions. Curr Psychiatry Rep. 2014 Nov;16(11):506. PMID:25218604

9. Abegunde DO, Mathers CD, Adam T, Ortegon M, Strong K. The burden and costs of chronic diseases in low-income and middle-income countries. Lancet. 2007 Dec 8;370(9603):192938. PMID:18063029

10. Naylor C, Parsonage M, McDaid D, Knapp M, Fossey M, Galea A. Long-term conditions and mental health. The cost of comorbidities. London: The King's Fund and Centre for Mental Health; 2012.

11. World health statistics [Internet]. Global Health Observatory Data Repository, World Health Organization (http://apps. who.int/ghodata/?vid=720, accessed 18 June 2015).

12. Edwards TM, Švab I, Ivbijaro G, Scherger J, Clarke DD, Kellenberg GA. Multimorbidity in primary care mental health. In: Ivbijaro G, editor. Companion to Primary Care Mental Health. London: Radcliffe Publishing; 2012. pp. 672-68.

13. Patel V, Belkin GS, Chockalingam A, Cooper J, Saxena S, Unützer J. Grand challenges: integrating mental health services into priority health care platforms. PLOS Medicine 2013;10(5):1-6 e1001448.

14. Sibbald B, Shen J, McBride A. Changing the skill-mix of the health care workforce. J Health Serv Res Policy. 2004 Jan;9 Suppl 1:28-38. PMID:15006226

15. Dubois C-A, Singh D. From staff-mix to skill-mix and beyond: towards a systemic approach to health workforce management. Hum Resour Health. 2009;7:87. PMID:20021682

16. Fulton BD, Scheffler RM, Sparkes SP, Auh EY, Vujicic M, Soucat A. Health workforce skill mix and task shifting in low income countries: a review of recent evidence. Hum Resour Health. 2011;9:1-11. PMID:21223546
17. Buttorff C, Hock RS, Weiss HA, Naik S, Araya R, Kirkwood BR, et al. Economic evaluation of a task-shifting intervention for common mental disorders in India. Bull World Health Organ. 2012 Nov 1;90(11):813-21. PMID:23226893

18. McInnis MG, Merajver SD. Global mental health: Global strengths and strategies Task-shifting in a shifting health economy. Asian J Psychiatr. 2011 Sep;4(3):165-71. PMID:23051112

19. Scheffler RM, Bruckner TA, Fulton TA, Yoon J, Shen G, Chisholm D, et al. Human Resources for Mental Health: Workforce Shortage in Low and Middle Income Countries. Berkeley (CA): University of California; 2010.

20. Perry HB, Zulliger R, Rogers MM. Community health workers in low-, middle-, and high-income countries: an overview of their history, recent evolution, and current effectiveness. Annu Rev Public Health. 2014;35:399-421. PMID:24387091

21. Patel V, Weiss HA, Chowdhary N, Naik S, Pednekar S, Chatterjee $\mathrm{S}$, et al. Lay health worker led intervention for depressive and anxiety disorders in India: impact on clinical and disability outcomes over 12 months. Br J Psychiatry. 2011 Dec;199(6):459-66. 10.1192/bjp.199.6.A23 PMID:22130747

22. Integrating mental health into primary care: a global perspective. Geneva: World Health Organization and World Organization of Family Doctors; 2008 (http://www.who.int/mental_health/resources/mentalhealth_PHC_2008.pdf, accessed 18 June 2015).

23. Collins C, Hewson DL, Munger R, Wade T. Evolving models of behavioural health integration in primary care. New York (NY): Milbank Memorial Fund; 2010.

24. Guidance and competences for the provision of services using practitioners with special interest (PwSIs) in mental health. London: Department of Health, Royal College of General Practitioners, Royal Pharmaceutical Society of Great Britain, NHS Primary Care Contracting; 2009.

25. London Health Programmes 1. Mental health services case for change for London. London: National Health Service; 2010 (http:// www.londonhp.nhs.uk/wp-content/uploads/2011/03/1.-Casefor-change-low-res.pdf, accessed 18 June 2015).

26. London Health Programmes. 2. Mental health models of care for London. London: National Health Service; 2010 (http://www. londonhp.nhs.uk/wp-content/uploads/2011/03/2.-Models-ofcare-low-res.pdf, accessed 18 June 2015).

27. Marmot M. Fair society, healthy lives: the Marmot review. London: UCL Institute of Health Equity; 2010.

28. The determinants of effective integration of health and social care. Cardiff, Wales: Health and Wellbeing Best Practice and Innovation Board; 2013 (http://www2.nphs.wales.nhs.uk:8080/ externaldocs.nsf/85c50756737f79ac80256f2700534ea3/714 d0d6339a19c1180257b180058a29d/\$FILE/The\%20Determinants\%20of\%20Effective $\% 20$ Integration $\% 20$ of $\% 20$ Health $\% 20$ and\%20Social\%20Care\%20FINAL.pdf, accessed 18 June 2015).

29. Integrated care pilots: final report, summary version. Cambridge, England: RAND Europe, Ernst and Young LLP, for the Department of Health; 2012 (https://www.gov.uk/government/uploads/system/uploads/attachment_data/file/146776/ dh_133126.pdf, accessed 18 June 2015).

30. Aims, concept and structure of the book. In: Ivbijaro G, editor. Companion to primary care mental health. London: Radcliffe Publishing; 2012:4.

Continued from page 406

${ }^{6}$ Marital and Family Therapy Program, University of San Diego, San Diego, California, United States of America. ${ }^{7}$ Waltham Forest Town Hall, London, United Kingdom. ${ }^{8}$ East London NHS Foundation Trust, London, United Kingdom. 